\title{
Atomically thin optical lenses and gratings
}

\author{
Jiong Yang ${ }^{1} \dagger$, Zhu Wang ${ }^{2} \dagger$, Fan Wang ${ }^{3}$, Renjing Xu ${ }^{1}$, Jin Tao ${ }^{1}$, Shuang Zhang ${ }^{1}$, Qinghua Qin ${ }^{1}$, \\ Barry Luther-Davies ${ }^{4}$, Chennupati Jagadish ${ }^{3}$, Zongfu $\mathrm{Yu}^{2 *}$ and Yuerui $\mathrm{Lu}^{1 *}$
}

Two-dimensional (2D) materials have emerged as promising candidates for miniaturized optoelectronic devices due to their strong inelastic interactions with light. On the other hand, a miniaturized optical system also requires strong elastic light-matter interactions to control the flow of light. Here we report that a single-layer molybdenum disulfide $\left(\mathrm{MoS}_{2}\right)$ has a giant optical path length (OPL), around one order of magnitude larger than that from a single-layer of graphene. Using such giant OPL to engineer the phase front of optical beams we have demonstrated, to the best of our knowledge, the world's thinnest optical lens consisting of a few layers of $\mathrm{MoS}_{2}$ less than $6.3 \mathrm{~nm}$ thick. By taking advantage of the giant elastic scattering efficiency in ultra-thin high-index 2D materials, we also demonstrated high-efficiency gratings based on a single- or few-layers of $\mathrm{MoS}_{2}$. The capability of manipulating the flow of light in 2D materials opens an exciting avenue towards unprecedented miniaturization of optical components and the integration of advanced optical functionalities. More importantly, the unique and large tunability of the refractive index by electric field in layered $\mathrm{MoS}_{2}$ will enable various applications in electrically tunable atomically thin optical components, such as micro-lenses with electrically tunable focal lengths, electrical tunable phase shifters with ultra-high accuracy, which cannot be realized by conventional bulk solids.

Light: Science \& Applications (2016) 5, e16046; doi:10.1038/Isa.2016.46; published online 11 March 2016

Keywords: atomically thin; grating; micro-lens; $\mathrm{MoS}_{2}$; two-dimensional

\section{INTRODUCTION}

Interactions between light and matter can be divided into two categories: inelastic and elastic ${ }^{1}$. An inelastic interaction involves energy transfer between photons and electrons or phonons. In contrast, elastic interactions do not involve energy transfer and are responsible for controlling the propagation of light. Optical components, such as resonant cavities, waveguides, lenses, gratings, and, more recently, optical meta-materials ${ }^{2}$ and photonic crystals ${ }^{3}$, all rely on strong elastic interactions between light and matter to achieve sophisticated control of the flow of light. Strong elastic interactions rely on significant changes of the amplitude and phase of the light accumulated over a long optical path and, hence, for very thin materials, such as a twodimensional(2D) graphene sheet, the interaction is generally very small ${ }^{4}$. Considerable effort has been devoted to this issue, but success has been only achieved in the mid- to far-infrared where the plasmonic resonance in graphene can enhance the elastic optical response ${ }^{5-7}$. It remains a great challenge to manipulate the flow of light using atomically thin $2 \mathrm{D}$ materials in the important visible and near-infrared spectral regions where $2 \mathrm{D}$ materials have most interesting optoelectronic properties ${ }^{8-19}$. Rather surprisingly, as we will show later, the strength of the elastic interaction in a thin 2D material increases dramatically with increasing refractive index because of the unique geometry associated with an ultra-thin film. Such favorable scaling makes high-index transition-metal dichalcogenide (TMD) 2D semiconductors ${ }^{11,20-22}$, such as molybdenum disulfide $\left(\mathrm{MoS}_{2}\right)$, particularly attractive for strong elastic light-matter interactions.

\section{MATERIALS AND METHODS}

Device fabrication and characterization

For the phase-shifting interferometry (PSI) measurements, single- and few-layer TMD semiconductors and graphene were deposited onto a $\mathrm{SiO}_{2} / \mathrm{Si}$ substrate $\left(275 \mathrm{~nm}\right.$ thermal $\mathrm{SiO}_{2}$ ) by mechanical exfoliation using $3 \mathrm{M}$ scotch tape. All Raman and photoluminescence (PL) measurements were conducted with a Horiba Jobin Yvon T64000 microRaman/PL system, with a $532 \mathrm{~nm}$ Nd:YAG green laser for excitation. All the optical path length (OPL) characterizations were obtained using a Veeco NT9100 phase-shifting interferometer. The atomically thin micro-lens and gratings were fabricated in a FEI Helios 600 NanoLab focused ion beam (FIB) system (Gallium ion source) using pre-calibrated dosage, optimized beam voltage $(30 \mathrm{kV})$ and beam current $(9.7 \mathrm{pA})$. The gratings and micro-lens were characterized using a green laser with a wavelength of $532 \mathrm{~nm}$.

\section{Numerical simulation}

Rigorous coupled-wave analysis (RCWA) was used to calculate the phase delay and grating efficiency. The method numerically solves Maxwell's equations in multiple layers of structured materials by expanding the field in Fourier space. The finite element method was

${ }^{1}$ Research School of Engineering, College of Engineering and Computer Science, the Australian National University, Canberra, ACT 2601, Australia; ${ }^{2}$ Department of Electrical and Computer Engineering, University of Wisconsin, Madison, WI 53706, USA; ${ }^{3}$ Department of Electronic Materials Engineering, Research School of Physics and Engineering, the Australian National University, Canberra, ACT 2601, Australia and ${ }^{4}$ CUDOS, Laser Physics Centre, Research School of Physics and Engineering, the Australian National University, Canberra, ACT 2601, Australia

†These authors contributed equally to this work.

Correspondence: ZF Yu, Email: zyu54@wisc.edu; YR Lu, Email: yuerui.lu@anu.edu.au

Received 5 August 2015; revised 10 November 2015; accepted 17 November 2015; accepted article preview online 18 November 2015 
used to calculate the optical scattering cross-section of the nano ribbons.

\section{RESULTS AND DISCUSSION}

Refractive optical components rely on the OPL to modify the phase front of an optical beam. The OPL is directly related to the geometrical length of the light path. As a result, it is normally expected that the OPL of a monolayer of a 2D material would be too small to have a significant impact on the phase front because the layer is so thin. However, here we measured a giant OPL of $38 \mathrm{~nm}$ from a single-layer $\mathrm{MoS}_{2}$, which is more than 50 times larger than its physical thickness of $0.67 \mathrm{~nm}$ and around one order of magnitude larger than the measured OPL of a single-layer graphene that was found to be only $4.4 \mathrm{~nm}$ (Figure 1).

In our experiments, single- or few-layer $\mathrm{MoS}_{2}$ flakes were transferred onto a silicon wafer with $275 \mathrm{~nm}$ of surface thermal oxide by mechanical exfoliation ${ }^{4,7}$. The flakes were firstly identified by their optical contrast in an optical microscope. Regions with different colors corresponded to $\mathrm{MoS}_{2}$ flakes with different thicknesses (Figure 1a). Due to their high refractive index, these atomically thin $\mathrm{MoS}_{2}$ layers have significant and layer-dependent OPL values and this enables the layers to be easily identified by phase-shifting interferometry (PSI) (Figure $1 \mathrm{~b}$ and 1c). PSI is capable of measuring the vertical OPL to an accuracy of around $0.1 \mathrm{~nm}$, by analyzing the digitized interference pattern obtained during a well-controlled phase shift (Supplementary Fig. S1 and S2). The measured OPL value of the $\mathrm{MoS}_{2}$ flake on $\mathrm{SiO}_{2}$ substrate at $535 \mathrm{~nm}$ was determined by:

$$
\mathrm{OPL}_{\mathrm{MoS}_{2}}=-\frac{\lambda}{2 \pi}\left(\phi_{\mathrm{MoS}_{2}}-\phi_{\mathrm{SiO}_{2}}\right)
$$

where $\lambda$ is the wavelength of the light source, $\phi_{\mathrm{MoS}_{2}}$ and $\phi_{\mathrm{SiO}_{2}}$ are the PSI-measured phase shifts of the light reflected from the $\mathrm{MoS}_{2}$ flake and the $\mathrm{SiO}_{2}$ substrate (Figure 1d inset), respectively. We characterized multiple samples and obtained statistical values of the OPL for single- and few-layer $\mathrm{MoS}_{2}$ samples as shown in Figure 1d. For each number of layers of $\mathrm{MoS}_{2}$, at least five different samples were characterized in these statistical measurements. The layer number could be quickly determined by the measured layer-dependent OPL values and the deduced layer number was confirmed by corresponding atomic force microscopy (AFM) images (Figure 1e and 1f); Raman microscopy (Supplementary Fig. S3a); and PL measurements (Supplementary Fig. S3b) on the same samples. For comparison, we performed the same characterizations on mechanically exfoliated graphene samples (Figure $1 \mathrm{~d}$ and Supplementary Fig. S4, S5, and S6). The measured average and standard deviation error of the OPL values from $1 \mathrm{~L}, 2 \mathrm{~L}, 3 \mathrm{~L}$, and $4 \mathrm{~L} \mathrm{MoS}_{2}$ samples were $38.0 \pm 2.8 \mathrm{~nm}, 85.4 \pm 2.2 \mathrm{~nm}, 124.0 \pm 6.6 \mathrm{~nm}$, and $162.6 \pm 9.0 \mathrm{~nm}$, respectively, while those from $1 \mathrm{~L}, 2 \mathrm{~L}, 3 \mathrm{~L}$, and $4 \mathrm{~L}$ graphene samples were $4.4 \pm 0.8 \mathrm{~nm}, 8.2 \pm 2.0 \mathrm{~nm}, 13.0 \pm 3.2 \mathrm{~nm}$, and $17.2 \pm 3.6 \mathrm{~nm}$, respectively, indicating that $\mathrm{MoS}_{2}$ has an OPL per layer approximately an order of magnitude larger than graphene.

This giant OPL is created by relatively strong multiple reflections at the air- $\mathrm{MoS}_{2}$ and $\mathrm{MoS}_{2}-\mathrm{SiO}_{2}$ interfaces. We consider a simple interface between air and $\mathrm{SiO}_{2}$, each occupying half infinite space. A layer of $2 \mathrm{D}$ material with a real refractive index $n$ is placed in between the two media. The high impedance mismatch at these interfaces leads to large reflection coefficients, which cause the strong multiple reflections of light in the 2D material(Figure 2a). The amplitude of the reflected light is the summation of the multiple reflections off the interfaces of the thin high index layer $R_{i}$, where $i$ indicates the number of round trips in the $2 \mathrm{D}$ material. As the index increases so does the reflectivity of the interfaces, which increases the effective number of transits of the light through the high index layer and thus the OPL of the reflected light (Figure 2a). We verify this intuition with numerical calculation as shown by the dashed line in Figure 2c. The magnitude of OPL difference comparing with and without the $2 \mathrm{D}$ material on $\mathrm{SiO}_{2}$ (Supplementary Fig. S7) increases rapidly with increasing $n$. The OPL is low for low-index 2D materials, where the small reflection coefficients cause $R_{i}$ to be small. This situation in illustrated schematically in Figure $2 \mathrm{~b}$. Additionally, in the experiment, we used a silicon substrate with a layer of $275 \mathrm{~nm}$ thermal $\mathrm{SiO}_{2}$ on its surface, which forms a weak Fabry-Perot resonance. As a result of this weak resonant enhancement, the OPL is further enhanced by a factor of around 1.5 as shown by the solid line in Figure 2c. Figure 2c also shows the OPL for a few other materials. The OPL of high-index 2D materials, such as $\mathrm{MoS}_{2}$, is remarkably larger than that of $\mathrm{SiO}_{2}$, graphene, $\mathrm{Au}$ or $\mathrm{Si}$. The wavelength used for these calculations was $535 \mathrm{~nm}$. The refractive indices used for single-layer $\mathrm{MoS}_{2}{ }^{23}$, silicon, graphene ${ }^{24}, \mathrm{SiO}_{2}$, and Au were $4.4+0.6 i, 4.15+0.044 i, 2.6+1.3 i, 1.46$, and $0.47+2.4 i$, respectively. In order to approximate our simulation to the actual condition, for $2 \mathrm{~L}$ $\mathrm{MoS}_{2}$, the refractive index in our simulation is assumed to be $4.4+0.6 i$ (the index for $1 \mathrm{~L} \mathrm{MoS}_{2}$ ); for $3 \mathrm{~L}$ and higher layer numbers, the refractive index is assumed to be $5.2+1.1 i$ (the index for bulk $\left.\mathrm{MoS}_{2}\right)^{23}$. In addition, it should be noted that the giant OPL is not a narrow band effect. The calculated OPL for $1 \mathrm{~L} \mathrm{MoS}_{2}$ is above $20 \mathrm{~nm}$ at the wavelength ranging from $450 \mathrm{~nm}$ to $560 \mathrm{~nm}$ (Supplementary Fig. S8). The spectral position for highest OPL can be adjusted by changing the thickness of the $\mathrm{SiO}_{2}$.

Even more remarkably, the OPL of single-, bi-, triple-, and quadruple-layer $\mathrm{MoS}_{2}$ scales almost linearly with the number of layers, offering the exciting opportunity of controlling the OPL using the number of layers of $\mathrm{MoS}_{2}$. When the layer thickness increases by $1 \mathrm{~nm}$, the OPL increases by over $50 \mathrm{~nm}$. Such a rapid change of OPL with thickness allows us to control the phase front of an optical beam very effectively using only an atomically thin structure. The theoretical and numerical predictions (Figure $2 \mathrm{~d}$ ) were well supported by the experimental data as shown in Figure 1d.

Next, we demonstrate phase-front engineering by fabricating the world's thinnest lens based on a few atomic layers of $\mathrm{MoS}_{2}$ (Figure 3). We started with a flake of uniform 9L $\mathrm{MoS}_{2}(6.28 \mathrm{~nm}$ in thickness, Supplementary Fig. S9) and then used FIB to mill a predesigned bowl-shaped structure $(20 \mu \mathrm{m}$ in diameter) into the flake (Figure $3 \mathrm{a}$ and $3 \mathrm{~b}$ ). The gradual change of $\mathrm{MoS}_{2}$ thickness, from the center to the edge, led to a continuous and curved OPL profile for an incident beam, and this served as an atomically thin (reflective) concave micro-lens (Figure 3c). Based on the measured OPL profile, the focal length $f$ of this $\mathrm{MoS}_{2}$ micro-lens was calculated to be $-248 \mu \mathrm{m}$ (Supplementary Fig. S10). In order to realize the precise design for this $\mathrm{MoS}_{2}$ micro-lens, we used the statistical calibration curve between the OPL values of $\mathrm{MoS}_{2}$ flakes and their layer numbers (Figure 3d). All the OPL values were measured by PSI and the layer numbers were confirmed by AFM. The OPL of $\mathrm{MoS}_{2}$ increased almost linearly with increasing the layer number when the layer number was less than five. We noticed the nonlinear response of OPL versus layer number when the layer number is more than five and this could be attributed to the fact that the single path absorption in $\mathrm{MoS}_{2}$ layer become more when the layer is thicker. Thus, the amplitude of the light will decrease faster in one round trip. When the amplitude of the light becomes very small after several roundtrips, its contribution to the OPL becomes negligible.

We used a far-field scanning optical microscopy (SOM) to characterize the fabricated $\mathrm{MoS}_{2}$ micro-lens (Supplementary Fig. S11). The SOM system used a green laser (at $532 \mathrm{~nm}$ ) that was focused onto 
a

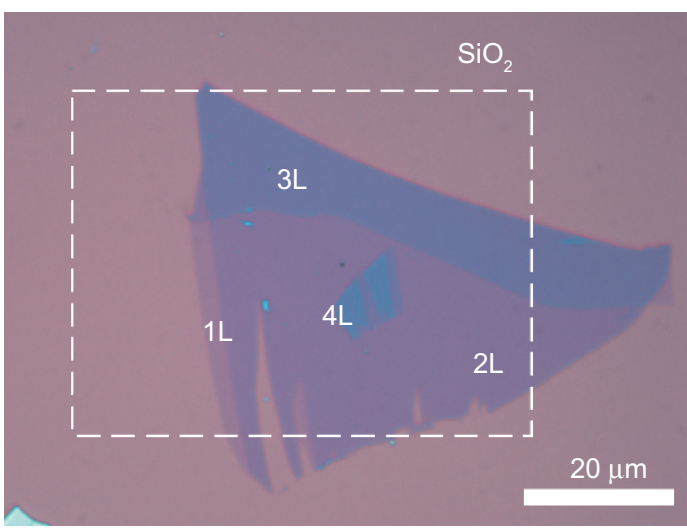

c

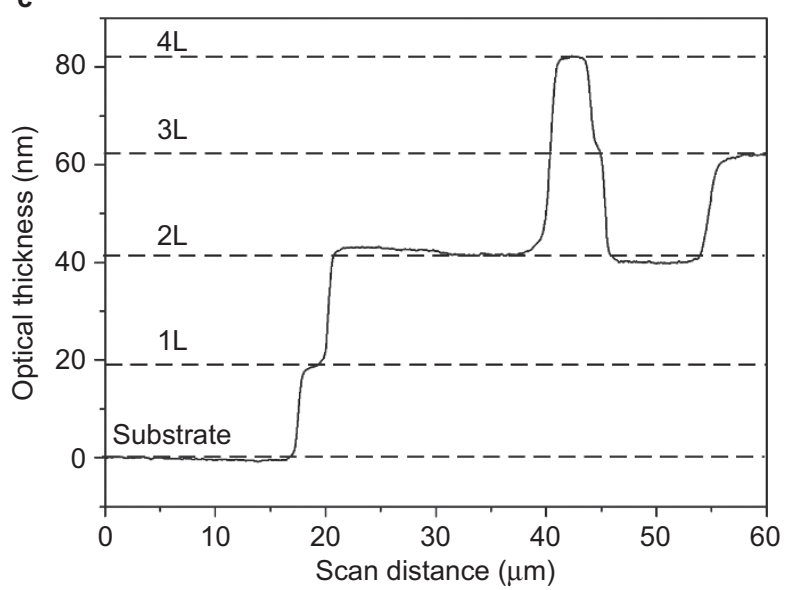

e

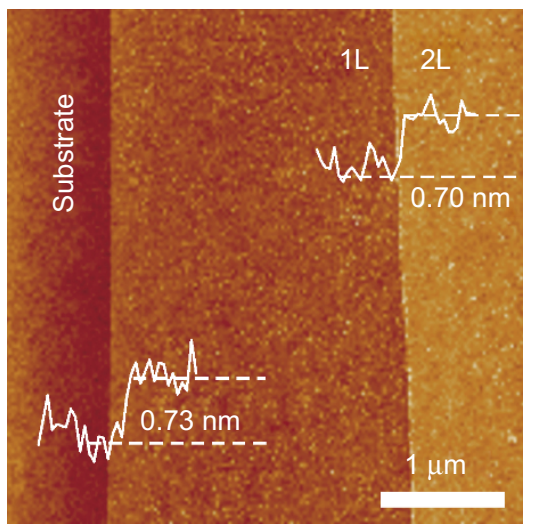

b

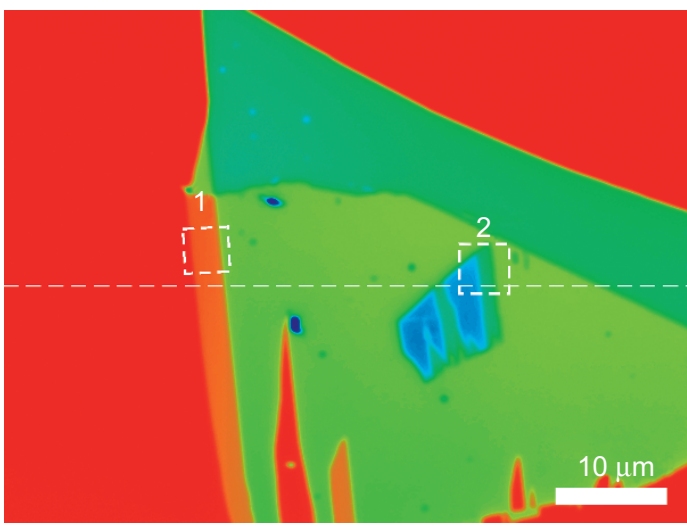

d

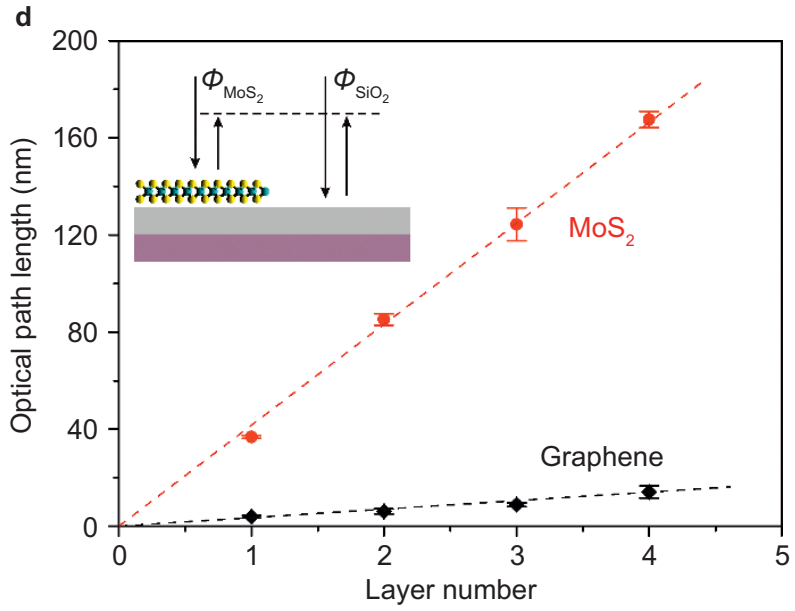

f

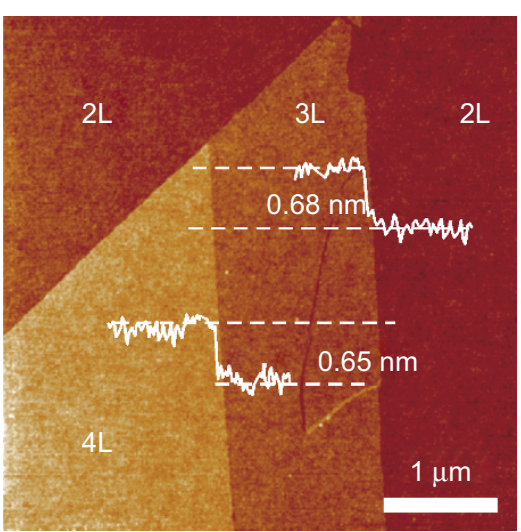

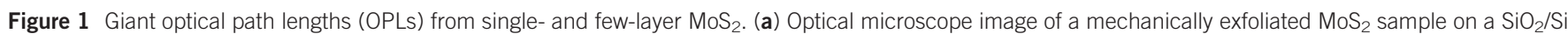

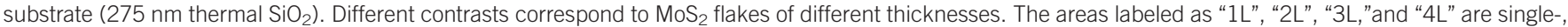

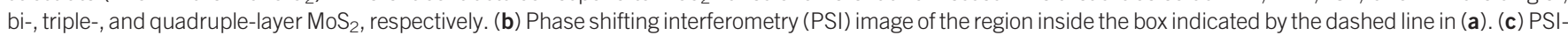

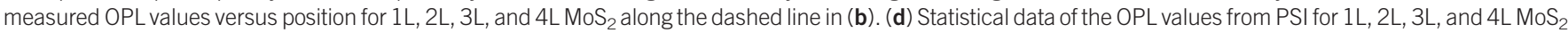

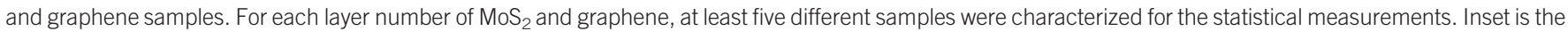

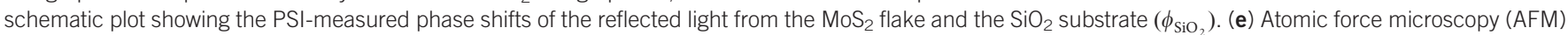
image of $1 \mathrm{~L}$ and $2 \mathrm{~L} \mathrm{MoS}_{2}$ from the box enclosed by the dashed line $1 \mathrm{in}$ (b). (f) AFM image of $3 \mathrm{~L}$ and $4 \mathrm{~L} \mathrm{MoS}$ from box enclosed by the dashed line 2 in (b).

the focal plane of an Olympus 10X (NA $=0.25$, depth of focus $18 \mu \mathrm{m})$ objective lens. The setup offered the best collection efficiency for light emitted from a small volume located around the focal plane. The micro-lens was moved along the $z$-axis in steps of $10 \mu \mathrm{m}$ by a piezoelectrically driven stage. The camera recorded a series of the intensity distributions (Supplementary Fig. S12) with the $\mathrm{MoS}_{2}$ micro-lens positioned at different $z$ values. A three-dimensional data set was generated by data processing and a cross-section profile was obtained along the $x$-and $z$-axes to illustrate the average distribution of the light intensity in these directions (Figure 3e). When the $\mathrm{MoS}_{2}$ micro-lens was placed at a distance $2|f|$ above the focal plane, the focused incident light would be exactly reimaged which is equivalent to the light coming from a point source (Supplementary Fig. S12d). Therefore, the camera recorded a well-focused light spot. The focal 
a

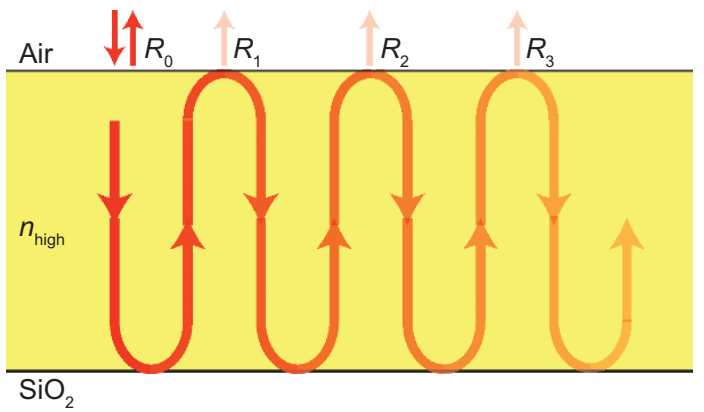

c

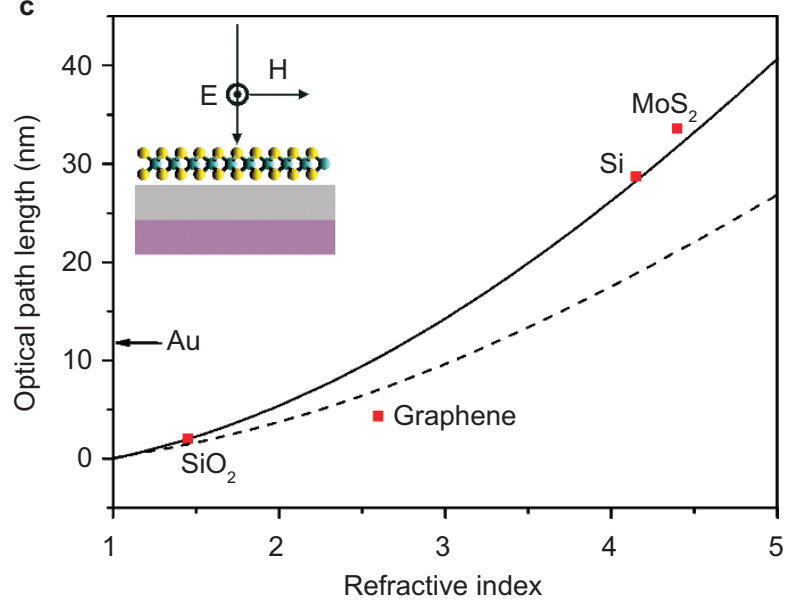

b

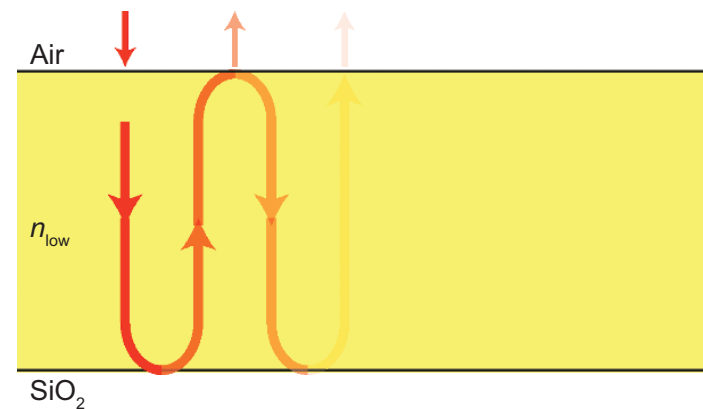

d

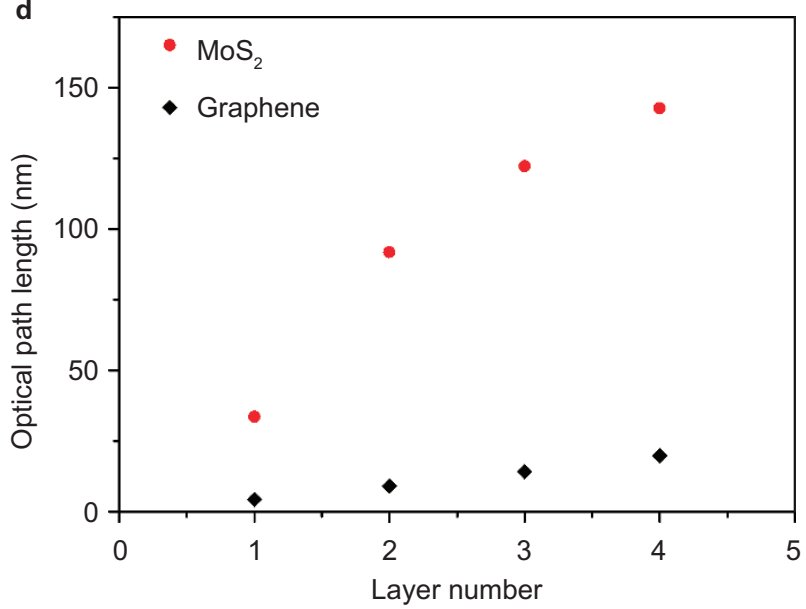

Figure 2 High refractive index enabled giant OPL in ultra-thin film. (a and $\mathbf{b}$ ) Schematic plots of multiple reflections at the interfaces of ultra-thin 2D materials. High refractive index leads to a large reflection coefficient. Light is reflected many times inside the material and leads to a highly enhanced light path, indicated as (a). For low refractive index material, the light path is much less enhanced because of the small reflection coefficient, indicated as (b). (c) Simulated OPL values for light reflected from $2 \mathrm{D}$ material $\left(0.67 \mathrm{~nm}\right.$ in thickness) with different indices on $\mathrm{S} \mathrm{SiO}_{2}(275 \mathrm{~nm}) / \mathrm{Si}$ substrate (solid line) and $\mathrm{SiO}_{2}$ substrate with infinite thickness (dashed line). The calculated OPLs of $0.67 \mathrm{~nm} \mathrm{Au}, 0.67 \mathrm{~nm} \mathrm{SiO}, 1 \mathrm{~L}\left(0.34 \mathrm{~nm}\right.$ ) graphene, and $1 \mathrm{~L}(0.67 \mathrm{~nm}) \mathrm{MoS}_{2}$ are represented by markers. (d) Simulated OPL values for $1 \mathrm{~L}, 2 \mathrm{~L}, 3 \mathrm{~L}$, and $4 \mathrm{~L} \mathrm{MoS}$ and graphene on $\mathrm{SiO}_{2}(275 \mathrm{~nm}) / \mathrm{Si}$ substrate, respectively. The wavelength used in the simulations was $535 \mathrm{~nm}$.

length $f$ of the $\mathrm{MoS}_{2}$ micro-lens was measured to be $-240 \mu \mathrm{m}$ $(2 f=-480 \mu \mathrm{m})$, which matched very well with the simulated value $(-248 \mu \mathrm{m})$ using the measured OPL profile of the micro-lens. For comparison, we also ran the same characterization by using a planar substrate without the $\mathrm{MoS}_{2}$ micro-lens, and obtained the intensity distribution shown in Figure $3 \mathrm{f}$ and Supplementary Fig. S13. The lensing effect is clearly demonstrated by comparing the difference between Figure $3 \mathrm{e}$ and $3 \mathrm{f}$. In addition, the measured focal length of the $\mathrm{MoS}_{2}$ micro-lens shows weak polarization dependence (Supplementary Fig. S14), due to the low anisotropic dielectric response of $\mathrm{MoS}_{2}$. This makes $\mathrm{MoS}_{2}$ suitable for ultra-thin optical elements.

The efficiency of light scattering is another critical parameter for advanced light manipulation. Devices that employ photonic band gaps $^{25}$, Anderson localization ${ }^{26}$, and light trapping such as with thinfilm solar cells all rely heavily on strong light scattering. Unfortunately, in typical 2D materials, such as graphene, the scattering efficiency is very small, making it impossible to rely on collective scattering of nanostructured graphene to achieve functionalities such as gratings. Here, we show that single- and few-layer structured $\mathrm{MoS}_{2}$ film have extraordinarily high scattering efficiency, enabled by the combination of high index in a thin structure. The scattering efficiency is determined by the strength of the electric field in the material. Normally, the electric field inside a bulk material, particularly a high-index material is much weaker than that of incident light because of the impedance mismatch. The boundary condition of Maxwell's equations requires the tangential component of the electric field to be continuous across any interface. Because the layer is thin, this condition indicates that the electrical field inside a $2 \mathrm{D}$ material is almost as strong as the tangential component of the incident field. As a result, there is a strong polarization $p=\epsilon_{0}\left(n^{2}-1\right) E_{0}$, where $E_{0}$ is the electric field of $s$-polarized incident light, $n$ is the index of the material and $\epsilon_{0}$ is the electric permittivity of free space. The scattering power is proportional to the $p^{2}$ and, therefore, scales roughly as $n^{4}$. This scaling rule greatly favors high-index materials and is again uniquely available in ultra-thin materials. In contrast, for nanoparticles, the scattering power is proportional to $\left(\frac{n^{2}-1}{n^{2}+2}\right)^{2}$, which does not increase appreciably with the refractive index ${ }^{28}$.

Here we use the finite element method to explicitly calculate the scattering efficiency of 2D ribbons by solving Maxwell's equations. Figure 4a shows the calculated scattering cross-section of an infinitely long ribbon ( $30 \mathrm{~nm}$ wide and $0.67 \mathrm{~nm}$ thick) in air for s-polarized light at normal incidence. The scattering cross-section has units of nanometers because the length of the ribbon is considered infinite. The scattering cross-section increases by orders of magnitude when the index increases by just a few times (Figure 4a). For example, the scattering cross-section of a single-layer $\mathrm{MoS}_{2}$ ribbon is around 670 times, 54 times, and 18 times of those in $0.67 \mathrm{~nm} \mathrm{SiO}_{2}$, a single-layer graphene, and $0.67 \mathrm{~nm}$ of gold, respectively. Metal is generally considered as one of the strongest scattering materials and it is important to note that $\mathrm{MoS}_{2}$ even displays much stronger light scattering than gold. Moreover, the angular response of the scattering cross-section is also 
a

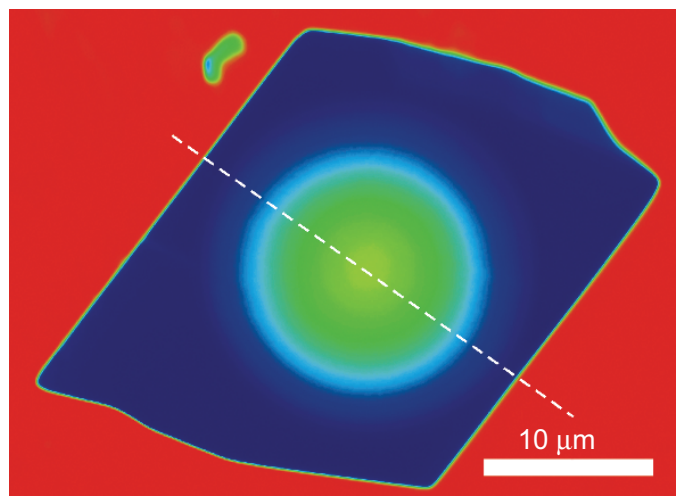

c

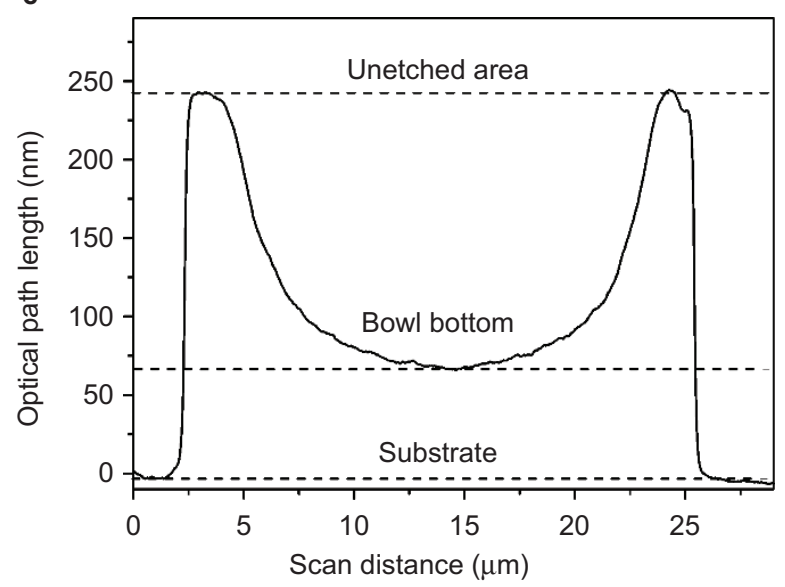

e

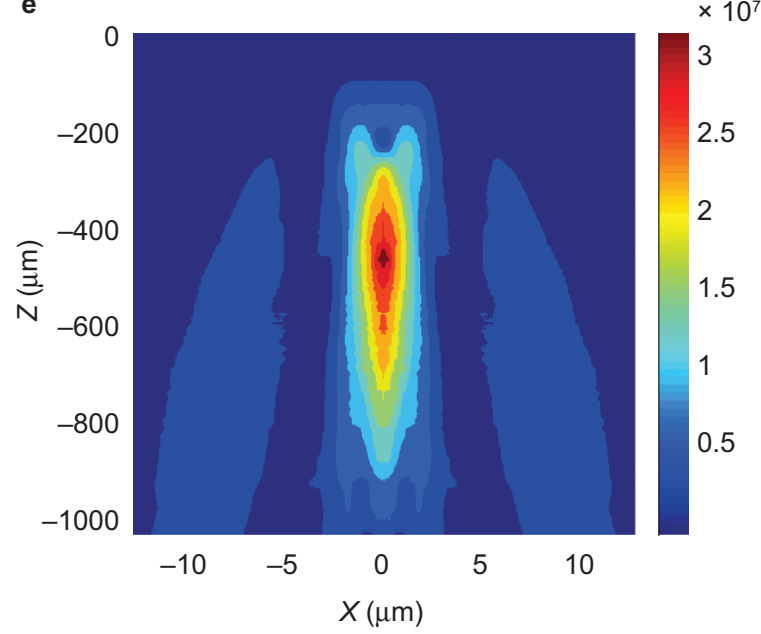

b

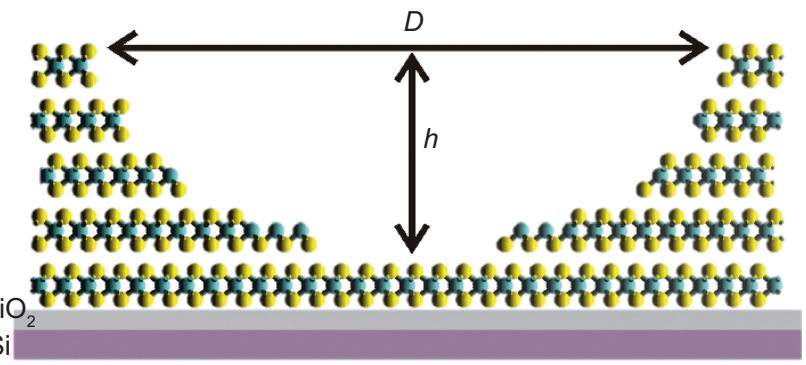

d

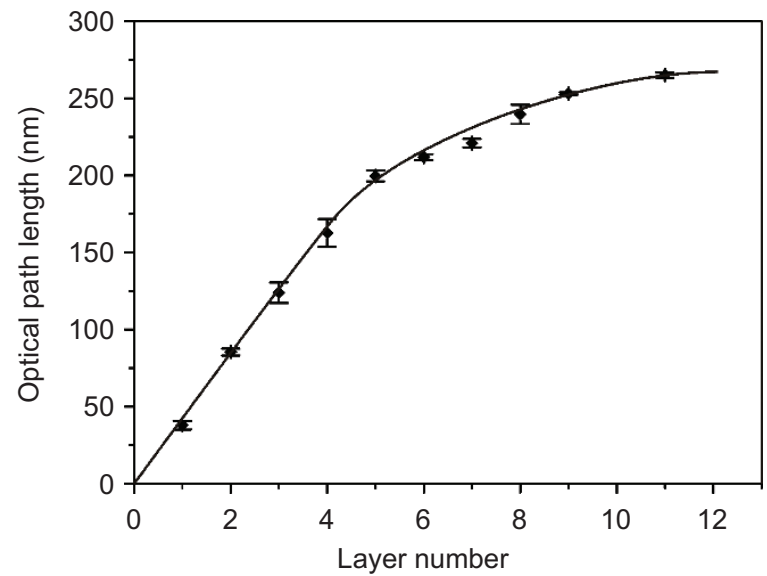

f

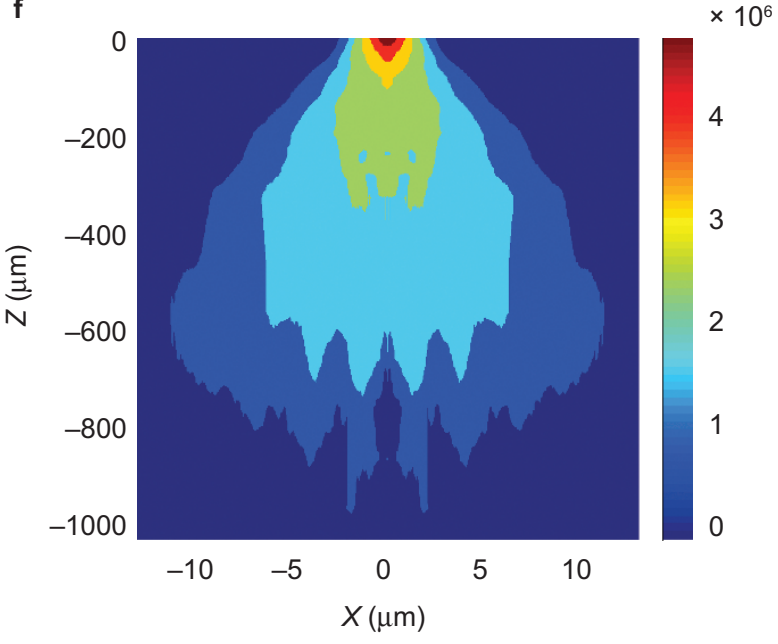

Figure 3 Atomically thin micro-lens fabricated from a few layers of $\mathrm{MoS}_{2}$. (a) PSI image of an atomically thin micro-lens fabricated on a 9L MoS 2 flake. (b) Schematic plot of the micro-lens structure. The bowl-shaped structure of the micro-lens was fabricated by FIB milling with atomic resolution in the vertical direction and sub-20 nm resolution in lateral direction. (c) Measured OPL values versus position for the direction indicated by the dashed line in (a). (d) Measured statistical data of the $\mathrm{OPL}$ values for $\mathrm{MoS}_{2}$ flakes with different layers ranging from $1 \mathrm{~L}$ to $11 \mathrm{~L}$. For each layer number of $\mathrm{MoS}_{2}$, at least five different samples were characterized in the statistical measurements. All the layer numbers were confirmed by AFM. (e) Intensity distribution pattern of the MoS $\mathrm{S}_{2}$ micro-lens measured by scanning optical microscopy (SOM). (f) Intensity distribution pattern of the planar reference $\mathrm{SiO}_{2} / \mathrm{Si}$ substrate measured by the same SOM setup.

isotropic (Supplementary Fig. S15). Such favorable scaling for high-index materials is uniquely available in ultra-thin materials. The giant scattering efficiency in high-index 2D materials makes it possible to achieve sophisticated light manipulation based on collective scattering by patterns of nanostructures. Next, we experimentally demonstrate efficient optical gratings made from only a few layers of atoms. Because of the giant scattering efficiency, the efficiency of $\mathrm{MoS}_{2}$ gratings is orders of magnitude greater than those made from conventional materials, such as $\mathrm{SiO}_{2}$ and gold, and other low-index 2D materials. 


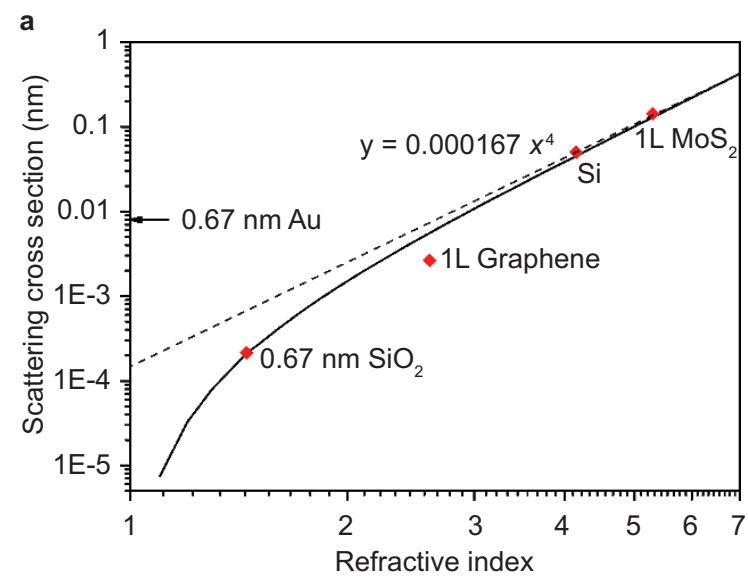

b

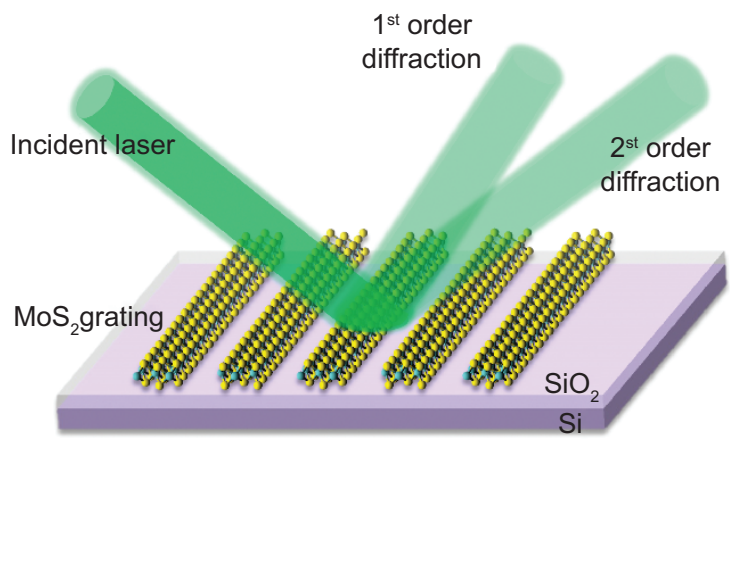

C

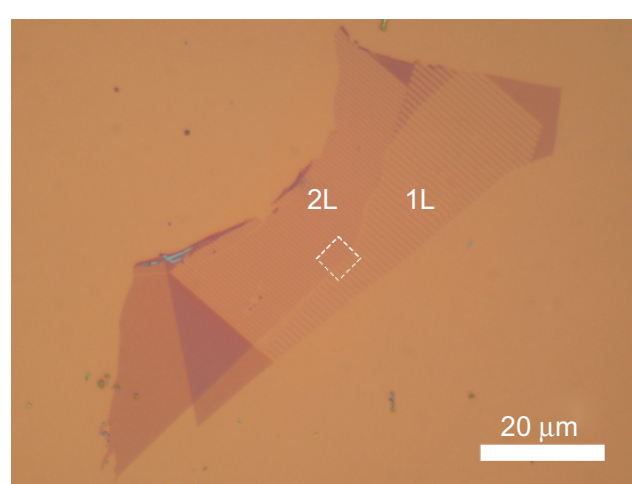

d

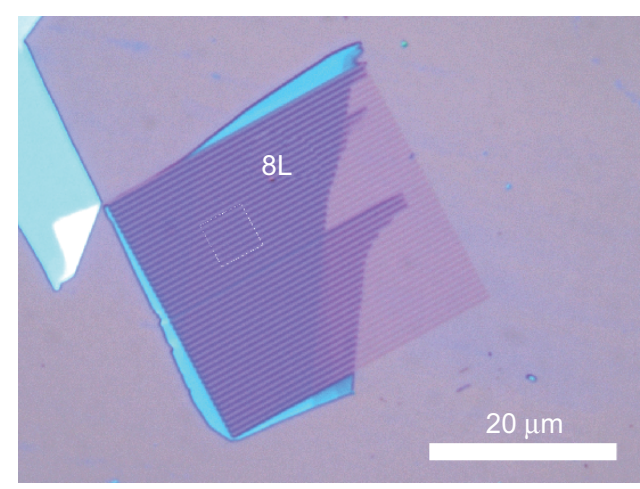

e

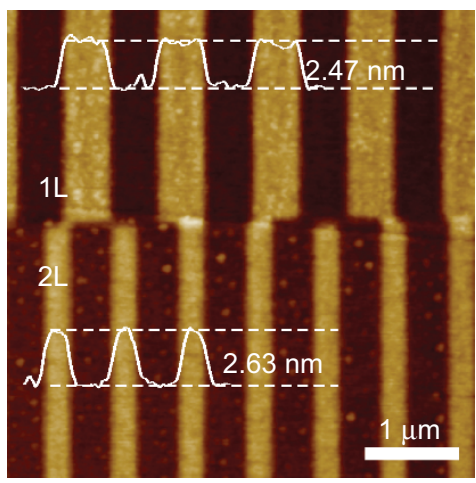

f

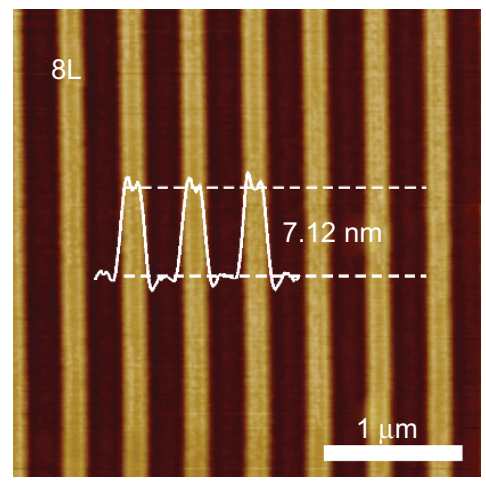

g

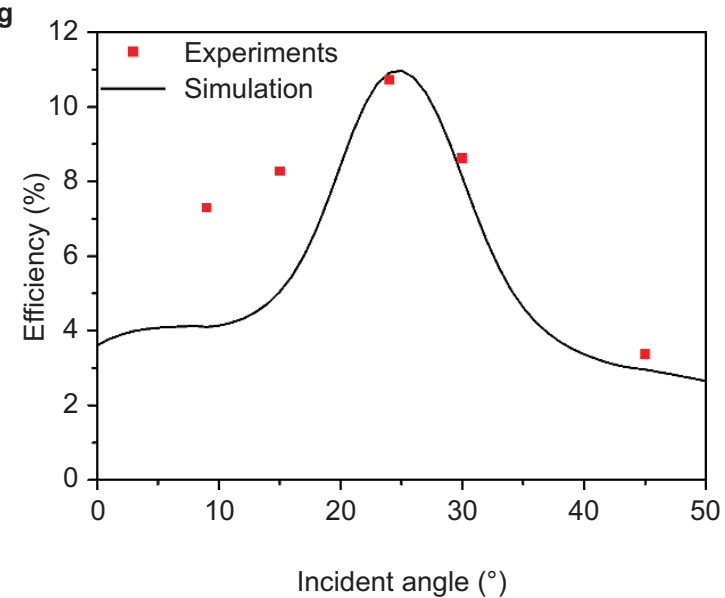

h

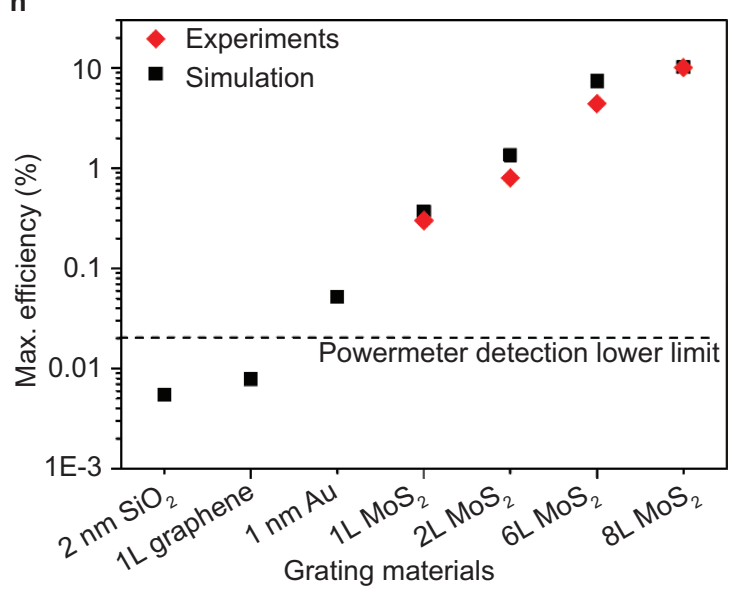


Figure 4 Atomically thin high-efficiency gratings made from a single- and a few-layers of $\mathrm{MoS}_{2}$. (a) Simulated scattering cross-section (SCS) versus refractive index for a layer of $0.67 \mathrm{~nm}$ thick material. The SCS values of $0.67 \mathrm{~nm} \mathrm{Au}, 0.67 \mathrm{~nm} \mathrm{SiO}, 1 \mathrm{~L}(0.34 \mathrm{~nm})$ graphene, and $1 \mathrm{~L}(0.67 \mathrm{~nm}) \mathrm{MoS}_{2}$ are represented by markers. The dashed line from equation $y=0.000167 x^{4}$ is added as a reference. (b) Schematic of the setup of the grating and for measurement of its diffraction efficiency. (c and $\mathbf{d}$ ) Optical microscope images of $1 \mathrm{~L}$ and $2 \mathrm{~L}$, and $8 \mathrm{~L} \mathrm{MoS}_{2}$ gratings. (e and $\left.\mathbf{f}\right) \mathrm{AFM}$ images of $1 \mathrm{~L}$ and $2 \mathrm{~L}$, and $8 \mathrm{~L} \mathrm{MoS}$ gratings. Note: based on the measured grating height, the $1 \mathrm{~L}, 2 \mathrm{~L}$, and $8 \mathrm{~L} \mathrm{MoS}_{2}$ were fully etched through and the $\mathrm{SiO}_{2}$ substrates underneath were over etched by around $1.5 \mathrm{~nm}$. From our control $\mathrm{SiO}_{2}$ grating experiments and from simulations, the grating contribution from this over etched $\mathrm{SiO}_{2}$ is negligible. (g) Simulated and measured efficiency of an $8 \mathrm{~L} \mathrm{MoS}_{2}$ grating versus incident angle of the light beam. (h) The comparison of the simulated and measured maximum grating efficiencies for different materials. The dash line represents the noise level of our light detection system, with the minimum detectable grating efficiency being $0.02 \%$.

We used FIB to mill grating patterns on $1 \mathrm{~L}, 2 \mathrm{~L}, 6 \mathrm{~L}$, and $8 \mathrm{~L} \mathrm{MoS}_{2}$ flakes (Figure 4 and Supplementary Fig. S16, S17, and S18). Grating parameters used in experiments, such as the periodicity and filling ratio, were based on optimal configuration predicted by simulations (Supplementary Table S1). The gratings were characterized using an $s$ polarized green laser (at a wavelength of $532 \mathrm{~nm}$ ). The laser beam has a diameter of around $200 \mu \mathrm{m}$, which was large enough to fully cover the grating. First-order and second-order diffraction beams were observed and the measured diffraction angles agreed with the predictions of the diffraction equation $d\left(\sin \theta_{\mathrm{d}}+\sin \theta_{\mathrm{i}}\right)=m \lambda$, where $\theta_{\mathrm{d}}$ and $\theta_{\mathrm{i}}$ are the diffraction angle and incident angle, respectively; $d$ is the period of the grating elements; and $m$ is an integer characterizing the diffraction order. The power of the first-order diffraction beam was measured and the grating efficiency $\eta$ was determined by $\eta=\left(P_{\mathrm{d}} / P_{\mathrm{i}}\right) *\left(S_{\mathrm{b}} / S_{\mathrm{g}}\right)$, where $P_{\mathrm{d}}$ and $P_{\mathrm{i}}$ were the measured powers of the diffracted and incident beams, respectively; $S_{\mathrm{b}}$ and $S_{\mathrm{g}}$ were the measured areas of the incident beam and the $\mathrm{MoS}_{2}$ grating, respectively. The measured grating efficiency is a function of the incident angle, which agrees well with our simulation (Figure $4 \mathrm{~g}$ ). The maximum grating efficiencies for the $1 \mathrm{~L}, 2 \mathrm{~L}, 6 \mathrm{~L}$, and $8 \mathrm{~L} \mathrm{MoS}_{2}$ gratings were measured to be $0.3 \%, 0.8 \%$, $4.4 \%$, and $10.1 \%$, respectively, which also agree well with the simulations (Figure 4h, Supplementary Table S1). For comparison, we also fabricated a grating from a graphene sheet deposited by large-area chemical vapor deposition (Supplementary Fig. S19a and S19b). The intensity of diffracted beam from the graphene grating was lower than the noise level of our light detection system, and thus had a maximum efficiency no greater than $0.02 \%$. From our simulations, the maximum grating efficiency of mono-layer graphene would be only $0.0078 \%$, which is around 47 times lower than that of a single-layer $\mathrm{MoS}_{2}$ grating. As another comparison, a $\mathrm{SiO}_{2}$ grating with $2 \mathrm{~nm}$ thickness was also fabricated (Supplementary Fig. S19c and S19d). Again no diffracted beam could be observed from the $\mathrm{SiO}_{2}$ grating due to the low grating efficiency in accordance with our numerical predictions (Figure 4h, Supplementary Table S1).

The efficiency of the $\mathrm{MoS}_{2}$ grating can be further improved by using a metallic mirror to replace the Si substrate. Based on simulations of optimized designs, the first-order grating efficiency of an 8L $\mathrm{MoS}_{2}$ grating can be up to $23.7 \%$ (Supplementary Table S2, Supplementary Fig. S20 and S21). In addition, an asymmetrical profile as used in high-efficiency gratings is expected to further improve the efficiency.

\section{CONCLUSIONS}

In conclusion, we have shown that high-index 2D materials have extraordinary elastic interactions with light, enabled uniquely by the ultrathin nature of $2 \mathrm{D}$ materials. As a result, wavefront shaping ${ }^{29,30}$ and efficient light scattering can be accomplished with atomically thin $2 \mathrm{D}$ materials, enabling a new class of optical components entirely based on high-index 2D materials. Moreover, compared to conventional diffractive optical components, the spatial resolution of phase-front shaping is much smaller than the wavelength, and is only limited by the nano-fabrication resolution, making it possible to eliminate undesired diffraction orders ${ }^{30} 2 \mathrm{D}$ materials also offer many unique advantages. Firstly, the extremely uniform thickness and the prefect surfaces with atomic roughness in layered high-index 2D materials provide us fantastic ways to precisely control the phase front of a wave. Secondly, the unique and large tunability of the refractive index by electric field ${ }^{31}$ in layered $\mathrm{MoS}_{2}$ will enable various applications in electrically tunable atomically thin optical components, such as micro-lenses with electrically tunable focal lengths, electrical tunable phase shifters with ultrahigh accuracy, which cannot be realized by conventional bulk solids. Thirdly, we also observed similar giant OPL in other TMD family $\mathrm{YX}_{2}$ $\left(\mathrm{Y}=\mathrm{Mo}, \mathrm{W} ; \mathrm{X}=\mathrm{S}, \mathrm{Se}, \mathrm{Te}\right.$ ) semiconductors, such as $\mathrm{WS}_{2}$ and $\mathrm{WSe}_{2}$ (Supplementary Fig. S22). The availability of different functional materials offers rich opportunities for the combination of optical and electronic properties, such as stacked atomically thin heterostructures for 2D optoelectronics. Fourthly, high-quality 2D TMD semiconductors can be deposited directly onto (or transferred to) various substrates with large size by chemical vapor deposition at low-cost ${ }^{32}$, potentially enabling low-cost flexible optical components. Lastly, quasi-2D optical components represents a significant advantage in manufacturing compared to conventional 3D optical components because different functionalities can all be achieved in a $2 \mathrm{D}$ platform sharing the same fabrication processes and this will greatly facilitate the large-scale manufacturing and integration. In summary, our work here opens an exciting opportunity to use high-index $2 \mathrm{D}$ materials to control the flow of light.

\section{AUTHOR CONTRIBUTIONS}

Y R L and Z F Y designed the project; J Y, R J X, and S Z carried out sample mechanical exfoliation and microscope imaging; J Y carried out the OPL, Raman, and PL measurements, AFM imaging, grating and micro-lens fabrication, grating efficiency measurement, and micro-lens characterization, with partial assistance from Y R L; Z W and Z F Y conducted the simulations and grating/micro-lens designs; F W and C J built the optical characterization setup for gratings and micro-lens. B L-D set up the PSI measurement system and provided technical support for the OPL characterization. J T and Q H Q undertook data processing for the micro-lens images. All authors contributed to the manuscript.

\section{COMPETING FINANCIAL INTERESTS}

The authors declare that they have no competing financial interests.

\section{ACKNOWLEDGEMENTS}

We would like to acknowledge support from the ACT node of the Australian National Fabrication Facility (ANFF) and, particularly, the technical support on the FIB provided by Dr Li (Lily) Li. We also thank Professor Jin-cheng Zheng, from Xiamen University (China), for helpful discussions, and Professor Vincent Craig and A/Professor Lan Fu, from the Australian National University, for support on AFM and optical imaging. We acknowledge the financial support from an ANU PhD scholarship;the Office of Naval Research (USA) under grant number N00014-14-1-0300; the Australian Research Council (grant number DE140100805); and the ANU Major Equipment Committee. 
1 Weiner J, Ho P-T. Light-matter Interaction, Fundamentals and Applications, Vol. 1. John Wiley \& Sons, Inc.; 2003.

2 Engheta N, Ziolkowski RW. Metamaterials: Physics and Engineering Explorations. Wiley-IEEE Press; 2006.

3 Joannopoulos JD, Johnson SG, Winn JN, Meade RD. Photonic Crystals: Molding the Flow of Light, 2nd edn. Princeton and Oxford: Princeton University Press; 2008.

4 Bonaccorso F, Sun Z, Hasan T, Ferrari AC. Graphene photonics and optoelectronics. Nat Photonics 2010; 4: 611-622.

5 Lee SH, Choi M, Kim T-T, Lee S, Liu M et al. Switching terahertz waves with gatecontrolled active graphenemetamaterials. Nat Mater 2012; 11: 936-941.

6 Ju L, Geng BS, Horng J, Girit C, Martin M et al. Graphene plasmonics for tunable terahertz metamaterials. Nat Nanotechnol 2011; 6: 630-634.

7 Vakil A, Engheta N. Transformation optics using graphene. Science 2011; 332: 1291-1294.

8 Lopez-Sanchez O, Lembke D, Kayci M, Radenovic A, Kis A. Ultrasensitive photodetectors based on monolayer MoS 2 . Nat Nanotechnol 2013; 8: 497-501.

9 Liu CH, Chang YC, Norris TB, Zhong ZH. Graphenephotodetectors with ultrabroadband and high responsivity at room temperature. Nat Nanotechnol 2014; 9: 273-278.

10 Sundaram RS, Engel M, Lombardo A, Krupke R, Ferrari AC et al. Electroluminescence in single layer $\mathrm{MoS}_{2}$. Nano Lett 2013; 13: 1416-1421.

11 Splendiani A, Sun L, Zhang YB, Li TS, Kim J et al. Emerging photoluminescence in monolayer $\mathrm{MoS}_{2}$. Nano Lett 2010; 10: 1271-1275.

12 Wang SX, Yu HH, Zhang HJ, Wang AZ, Zhao MW et al. Broadband few-layer $\mathrm{MoS}_{2}$ saturable absorbers. Adv Mater 2014; 26: 3538-3544.

13 Yin XB, Ye ZL, Chenet DA, Ye Y, O'Brien K et al. Edge nonlinear optics on a MoS 2 atomic monolayer. Science 2014; 344: 488-490.

14 Zeng HL, Dai JF, Yao W, Xiao D, Cui XD. Valley polarization in $\mathrm{MoS}_{2}$ monolayers by optical pumping. Nat Nanotechnol 2012; 7: 490-493.

15 Mak KF, He KL, Shan J, Heinz TF. Control of valley polarization in monolayer $\mathrm{MoS}_{2}$ by optical helicity. Nat Nanotechnol 2012; 7: 494-498.

16 Zhang S, Yang J, Xu RJ, Wang F, Li WF et al. Extraordinary photoluminescence and strong temperature/angle-dependent Raman responses in few-layer phosphorene. ACS Nano 2014; 8: 9590-9596.

17 Yang J, Xu RJ, Pei JJ, Myint YW, Wang F et al. Optical tuning of exciton and trion emissions in monolayer phosphorene. Light Sci App/ 2015; 4: e312; doi:10.1038/ Isa.2015.85

18 Yang J, Lü TY, Myint YW, Pei JJ, Macdonald D et al. Robust excitons and trions in monolayer $\mathrm{MoTe}_{2}$. ACS Nano 2015; 9: 6603-6609.
19 Xu RJ, Yang J, Zhu Y, Yan H, Pei JJ et al. Layer-dependent surface potential of phosphorene and anisotropic/layer-dependent charge transfer in phosphorene-gold hybrid systems. Nanoscale 2016; 8: 129-135.

20 Wang QH, Kalantar-Zadeh K, Kis A, Coleman JN, Strano MS. Electronics and optoelectronics of two-dimensional transition metal dichalcogenides. Nat Nanotechnol 2012; 7: 699-712.

21 Radisavljevic B, Radenovic A, Brivio J, Giacometti V, Kis A. Single-layer $\mathrm{MoS}_{2}$ transistors. Nat Nanotechnol 2011; 6: 147-150.

22 Xu XD, Yao W, Xiao D, Heinz TF. Spin and pseudospins in layered transition metal dichalcogenides. Nat Phys 2014; 10: 343-350.

23 Zhang $\mathrm{H}$, Ma YG, Wan Y, Rong X, Xie Z et al.Measuring the refractive index of highly crystalline monolayer $\mathrm{MoS}_{2}$ with high confidence.Sci Rep 2015; 5: 8440.

24 Blake P, Hill EW, Castro Neto AH, Novoselov KS, Jiang D et al. Making graphene visible. Appl Phys Lett 2007; 91: 063124.

25 Yablonovitch E. Inhibited spontaneous emission in solid-state physics and electronics. Phys Rev Lett 1987; 58: 2059-2062.

26 John S. Strong localization of photons in certain disordered dielectric superlattices. Phys Rev Lett 1987; 58: 2486-2489.

27 Yu ZF, Raman A, Fan SH. Thermodynamic upper bound on broadband light coupling with photonic structures. Phys Rev Lett 2012; 109: 173901.

28 Jackson JD. Classical Electrodynamics,3rd edn. John Wiley \& Sons Ltd.; 1998.

29 Lin D, Fan P, Hasman E, Brongersma ML. Dielectric gradient metasurface optical elements. Science 2014; 345: 298-302.

30 Yu NF, Capasso F. Flat optics with designer metasurfaces. Nat Mater 2014; 13: 139150.

31 Santos EJG, Kaxiras E. Electrically driven tuning of the dielectric constant in $\mathrm{MoS}_{2}$ layers. ACS Nano 2013; 7: 10741-10746.

32 van der Zande AM, Huang PY, Chenet DA, Berkelbach TC, You YM et al. Grains and grain boundaries in highly crystalline monolayer molybdenum disulphide. Nat Mater 2013; 12: 554-561.

\section{(c) (i) (5) $\odot$ This work is licensed under a Creative Commons Attribution-}

cc) ${ }_{\mathrm{BY}} \mathrm{NC}$ ND NonCommercial-NoDerivs 4.0 Unported License. The images or other third party material in this article are included in the article's Creative Commons license, unless indicated otherwise in the credit line; if the material is not included under the Creative Commons license, users will need to obtain permission from the license holder to reproduce the material. To view a copy of this license, visit http://creativecommons.org/licenses/ by-nc-nd/4.0/ 